\title{
COMPUTATIONAL INVESTIGATION OF SPECTROSCOPIC PARAMETERS IN PUTATIVE SECONDARY STRUCTURE ELEMENTS FOR POLYLACTIC ACID AND COMPARISON WITH EXPERIMENT
}

\author{
IZABELLA IRSAI ${ }^{\mathrm{a}}$, ALEXANDRU LUPAN $^{\mathrm{a}}$, CORNELIA MAJDIK $^{\mathrm{a}}$, \\ RADU SILAGHI-DUMITRESCU ${ }^{\text {** }}$
}

\begin{abstract}
Putative elements of secondary, tertiary and quaternary structure were examined for polylactic acid chains, attempting a parallel with secondary structure elements known from protein biology and also attempting an estimate, based on accurate atomic-level calculations, of interaction energies between polylactic acid chains. Spectroscopic parameters were predicted for all types of structure examined, in an attempt to aid our on-going efforts in synthesis and characterization of polylactic acid variants.
\end{abstract}

Keywords: polylactic acid, secondary structure, NMR, EPR, DFT, semiempirical

\section{INTRODUCTION}

Poly(lactic acid) (PLA) as a biodegradable polymer has a tremendous potential in medical, pharmacological and environmental applications [1-4]. It degrades to nontoxic lactic acid which is naturally present in human body.

PLA homopolymer can crystallize in three polymorphs: $\alpha$ [5-9], $\beta$ $[8,10]$ and $Y[7,12]$. The crystal structures have been studied by $X$-ray method, the experiments show the presence of $10_{3}$ and $3_{1}$ helical chains of molecules $[6,11]$.

The equimolecular mixture of poly(L-lactic acid) (PLLA) and poly(Dlactic acid) (PDLA) enantiomers has another crystal modification known as the sc-form with $3{ }_{1}$ helices [13-19].

\footnotetext{
a Babeş-Bolyai University, Faculty of Chemistry and Chemical Engineering, 11, Arany Janos Street, RO-4000228 Cluj-Napoca, Romania

*Corresponding author: rsilaghi@chem.ubbcluj.ro
} 
The crystal structures were also analyzed by computational chemistry. The poly(lactic acid) polymorphs were studied by rotational isomeric state models [20, 21], molecular dynamics [9, 10], Monte Carlo models [21,22], molecular mechanics [9] and quantum chemical [23-25] simulations. It was found that neither a pre $10_{3}$ nor $3_{1}$ helix could fit the experimental data perfectly, suggesting a certain degree of disorder in the structure.

\section{RESULTS AND DISCUSSION}

Four secondary-types structure were optimized - helical structures $\left(\alpha, \pi, 10_{3}\right)$ and $\beta$-sheet - employing molecular mechanics, semiempirical, ab initio and density functional methods. The highest-level method (DFT/M062x) denotes that the $\alpha, \pi$ and $10_{3}$ structures have very similar energies, with $\pi$ slightly favored by values, this in a contrast with results obtained with less accurate semiempirical and empirical methods, which predict larger differences and other structures as favorites.

Figure 1 shows optimized geometries for models of polylalctic acid (PLA) employed in the present study. Decameric structures of L-lactic acid (PLLA) as well as of alternating D,L monomers (PDLLA) were employed. Details of these structures and their relative energies are described elsewhere. The energy in the case of PLLA are generally smaller than in the case of PDLLA, suggesting that such structures are more stable.


Figure 1. Graphical representation of polylactic acid geometries optimized by DFT 
The vibrational frequencies were computed for these optimized structures. In addition it was calculated the vibrational frequencies of the polylactic structure described by DeSantis.

The IR spectra shows characteristic bands mainly due to methylene and carboxylic $\mathrm{C}=\mathrm{O}$ bonds. A repeat unit of PLA consists of three skeletal bonds: $\mathrm{C}-\mathrm{O}, \mathrm{O}-\mathrm{C}_{\alpha}, \mathrm{C}_{\alpha}-\mathrm{C}$. For PLA the bands are assigned due to backbone bond stretching $\left(\mathrm{C}-\mathrm{O}, \mathrm{O}-\mathrm{C}_{\alpha}, \mathrm{C}-\mathrm{C}_{\alpha}\right)$, backbone bond angle bending $\left(\mathrm{O}-\mathrm{C}-\mathrm{C}_{\alpha}\right.$, $\left.\mathrm{C}-\mathrm{O}-\mathrm{C}_{\alpha}, \mathrm{O}-\mathrm{C}_{\alpha}-\mathrm{C}\right)$, for the side branches the bending $\left(\mathrm{O}-\mathrm{C}_{\alpha}-\mathrm{C}_{\beta}, \mathrm{C}-\mathrm{C}_{\alpha}-\mathrm{C}_{\beta}, \mathrm{C}_{\alpha^{-}}\right.$ $\mathrm{C}=\mathrm{O})$ and stretching $\left(\mathrm{C}_{\alpha}-\mathrm{C}_{\beta}, \mathrm{C}=\mathrm{O}\right)$.

Figure 2 shows the IR spectrum of polylactic acid prepared as described in the Methods section. It can be seen the bands of $\mathrm{C}=\mathrm{O}$ stretching $\left(1751,20 \mathrm{~cm}^{-1}\right)$, C-OC stretching $\left(1117,42 \mathrm{~cm}^{-1}\right)$ and $\mathrm{CH}$ bending and C-OC stretching $\left(1064,37 \mathrm{~cm}^{-1}\right)$.

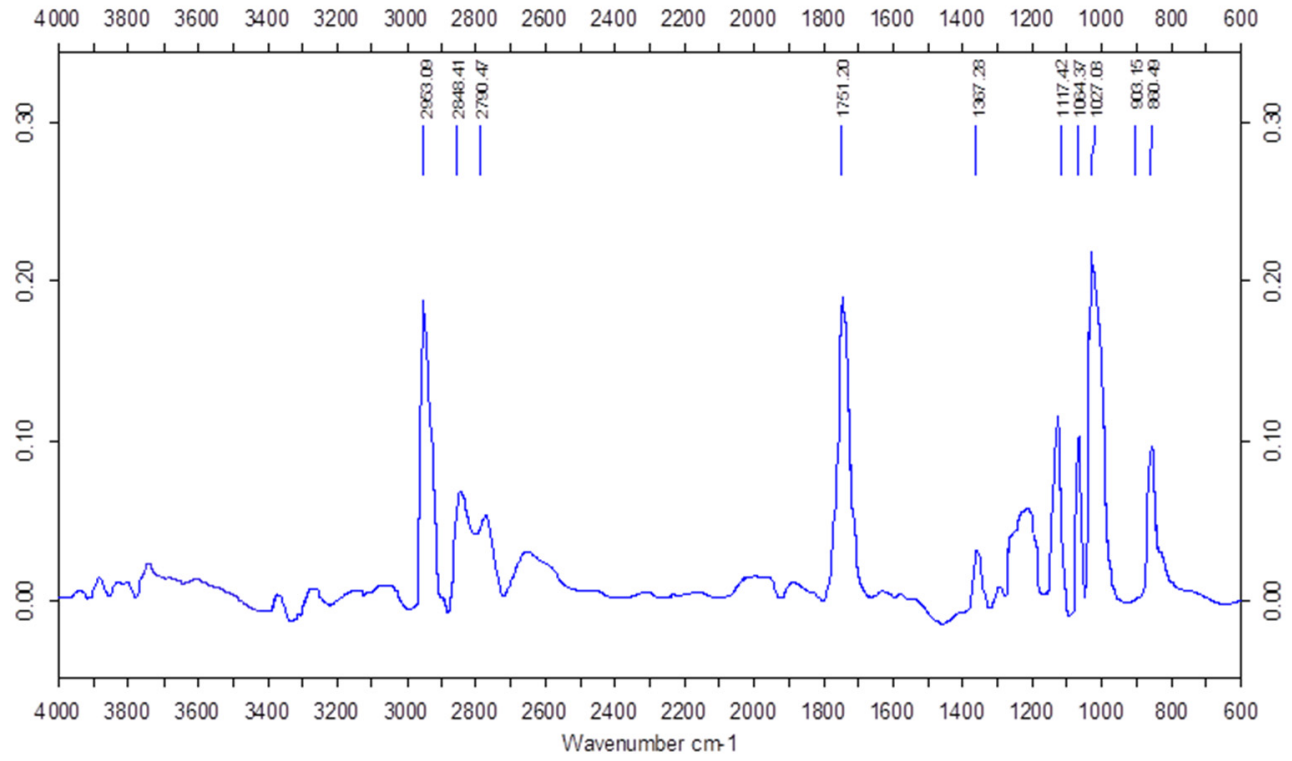

Figure 2. IR spectrum of poly(L-lactic acid) (cf. Materials and Methods )

Figure 3 shows computed IR spectra for the five secondary structure elements considered in the present work. The intensity of IR vibrations depend on the structure of the poly(lactic acid). 

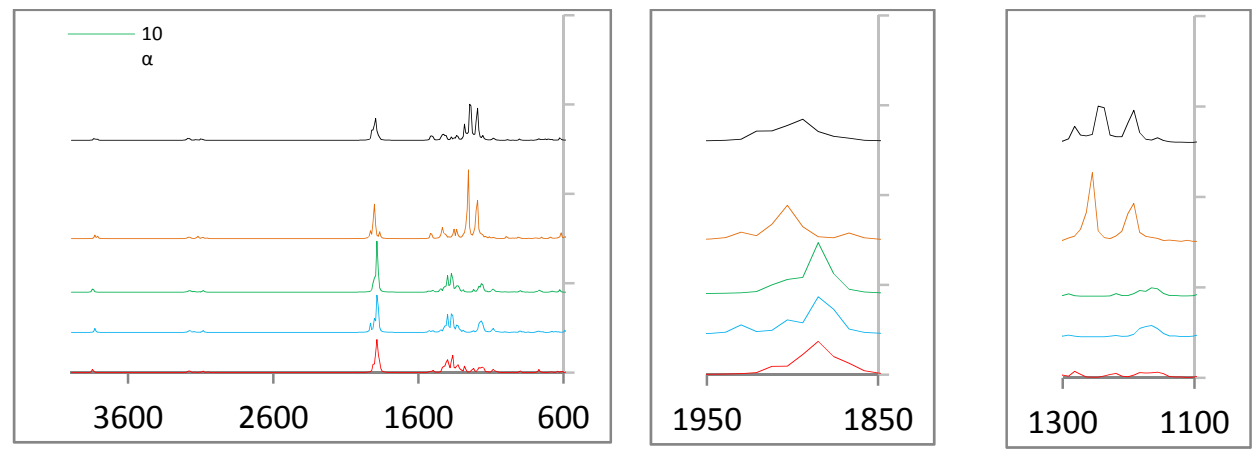

Figure 3. IR spectra of PLLA (M062x/6-31G**):(a) full spectrum;

(b) carbonyl stretching region; (c) backbone stretching region

The IR spectra of the five structures show the same number of bands. The solvation does not involve any additional band.

The bands below $225 \mathrm{~cm}^{-1}$ are mainly due to the skeletal torsion. The $225-925 \mathrm{~cm}^{-1}$ region bands are assigned due to the bending of the side branches. There are no significant intensities.

The $\mathrm{CH}_{3}$ is responsible for the appearance of the band in the 925$1110 \mathrm{~cm}^{-1}$ region due to the rocking vibrations. The solvation increases the intensities in the case of the three helical structures.

The $\mathrm{C}-\mathrm{CH}_{3}$ and $\mathrm{C}-\mathrm{OC}$ stretching cause the presence of bands in the $1124-1213 \mathrm{~cm}^{-1}$ backbone stretching region. The intensities of the bands corresponding to $\mathrm{C}_{-} \mathrm{CH}_{3}$ stretch are medium in the case of the helical structures, the water double these values. The $\beta$-sheet and the structure described by DeSantis provide weak bands. C-OC stretching band are shown in 1151-1217 $\mathrm{cm}^{-1}$ region. Assignment of the $\beta$-sheet is the most intensive, six times higher than the others. The solvation increases the intensity further. The structure described by DeSantis has a strong band in this region.

In the $1215-1412 \mathrm{~cm}^{-1}$ region it can be seen bands assigned to $\mathrm{CH}$, $\mathrm{CH}_{3}$ bending and $\mathrm{C}-\mathrm{O}-\mathrm{C}$ stretching. The intensity of the $\mathrm{CH}$ bending and $\mathrm{C}-$ $\mathrm{O}-\mathrm{C}$ stretching in the case of $\beta$-sheet and the structure described by DeSantis are very strong in contrast with the helical structure. These are the highest intensity bands in the entire spectrum. The water does not influence these intensities. The $\mathrm{CH}$ bending intensities are roughly the same with the exception of structure described by DeSantis. The solvation increases the 
intensities in the case of the helical structures. A different medium band appear in this region due to the $\mathrm{CH}$ bending and $\mathrm{CH}_{3}$ scissoring. It is worth noting the assignments in the $1407-1437 \mathrm{~cm}^{-1}$ region. In this interval appear the bands due to $\mathrm{CH}_{3}$ scissoring vibrations. In the case of the three helical structures are not significant intensities, but in the case of $\beta$-sheet the intensity is notable.

IR spectra of the PLLA in the range of $1865-1932 \mathrm{~cm}^{-1}$ appear to be distinct for each of the five conformations. There is not much difference between the $\mathrm{C}=\mathrm{O}$ stretching intensities calculated by DFT/M062x/6-31G** method. In turn the solvation increases the intensities in all five structures. The five conformers exhibit single absorption bands, spaced by about 9 and $18 \mathrm{~cm}^{-1}$ from each other.

The $\mathrm{CH}$ stretching, the symmetric and asymmetric stretching of $\mathrm{CH} 3$ result the appearance of weak bands in the 3050-3191 $\mathrm{cm}^{-1} \mathrm{CH}$ spectral region. It can be seen that the stretching frequencies are higher than the corresponding bending frequencies.

The calculated frequencies $\left(\mathrm{cm}^{-1}\right)$ and peak band assignment for the helical structures and $\beta$-sheet of PLLA are shown in tables 1-4.

In order to demonstrate that the chain conformation changes the interval of IR frequencies it was calculated the IR spectra of PDLLA and then compared to experimental data. Figure 4. shows the IR spectrum of PDLLA. The spectrum represent the bands of $\mathrm{CH}$ bending and $\mathrm{C}-\mathrm{OC}$ stretching $\left(1088 \mathrm{~cm}^{-1}\right), \mathrm{C}-\mathrm{OC}$ stretching $\left(1188 \mathrm{~cm}^{-1}\right), \mathrm{C}=\mathrm{O}$ stretching $\left(1752 \mathrm{~cm}^{-1}\right), \mathrm{CH}_{3}$ symmetric stretching $\left(2945 \mathrm{~cm}^{-1}\right), \mathrm{CH}_{3}$ asymmetric stretching $\left(2997 \mathrm{~cm}^{-1}\right)$ and OH stretching $\left(3737 \mathrm{~cm}^{-1}\right)$.

In the IR spectra of PDLLA it can be seen the same bands as in the case of PLLA. The bands below $900 \mathrm{~cm}^{-1}$ represent weak intensities. The $\mathrm{CH}_{3}$ rocking and $\mathrm{C}-\mathrm{CH}_{3}$ stretching band intensities decrease slightly compared to PLLA. The C-OC stretching's bands appear at lower values $(1146-1215 \mathrm{~cm}-1)$ than in the case of PLLA. The intensities of the $\alpha$ helix and the $\beta$-sheet increase significantly. The calculated intensity of assignment of $\beta$-sheet is bigger in vacuum than in the solvated model.

The $\mathrm{CH}$ bending and $\mathrm{C}-\mathrm{OC}$ stretching bands have equivalent values in the 1204-1325 $\mathrm{cm}^{-1}$ interval, but bigger than the PLLA's one; the $\beta$-sheet and the structure described by DeSantis bands' intensities are 5-8 higher than in the case of the helices. It was observed that intensities of $\beta$-sheet in vacuum and solvation show outstanding values over the others, but these values are much smaller than in the case of PLLA. 
Table 1. Peak band assignments for $\alpha-L-L A_{10}$

\begin{tabular}{|c|c|c|c|c|}
\hline \multicolumn{5}{|c|}{$\alpha-L-L A_{10}$} \\
\hline \multicolumn{2}{|c|}{ M062X 6-31G** } & \multicolumn{2}{|c|}{ M062X 6-31G** water } & \multirow[b]{2}{*}{ Assignment } \\
\hline $\mathrm{v}\left(\mathrm{cm}^{-1}\right)$ & Intensity & $\mathrm{v}\left(\mathrm{cm}^{-1}\right)$ & Intensity & \\
\hline $18-224$ & $0-6$ & $13-216$ & $0-9$ & skeletal torsion \\
\hline $233-296$ & $0-6$ & $224-266$ & $0-12$ & $\mathrm{CH}_{3}$ rocking \\
\hline $302-399$ & $0-48$ & 299-394 & $0-73$ & $\mathrm{CH}_{3}$ wagging \\
\hline 402 & 147 & 399 & 216 & $\mathrm{OH}$ (free) bending \\
\hline $407-411$ & $32-59$ & $401-405$ & $32-88$ & $\mathrm{CH}_{3}$ wagging \\
\hline 413 & 63 & 406 & 88 & $\mathrm{OH}$ (free) bending \\
\hline $416-558$ & $1-18$ & $409-592$ & $0-148$ & CCO bending \\
\hline 627 & 91 & 631 & 116 & $\mathrm{OH}(\mathrm{COOH})$ bending \\
\hline $663-789$ & $1-34$ & $663-787$ & $1-38$ & $\mathrm{C}=\mathrm{O}$ bending \\
\hline 848 & 7 & 841 & 11 & C-CO (carboxyl) stretching \\
\hline $879-909$ & $1-31$ & $880-908$ & $2-37$ & $\mathrm{CH}_{3}$ bending $+\mathrm{COC}$ bending \\
\hline $925-1105$ & $1-107$ & $924-1096$ & $1-238$ & $\mathrm{CH}_{3}$ rocking \\
\hline $1127-1164$ & $13-105$ & $1120-1155$ & $11-220$ & $\mathrm{C}-\mathrm{CH}_{3}$ stretching \\
\hline $1167-1189$ & $5-82$ & $1158-1182$ & $2-127$ & C-OC stretching \\
\hline $1218-1331$ & $1-118$ & $1217-1330$ & $31-228$ & $\begin{array}{l}\mathrm{CH} \text { bending }+\mathrm{C}-\mathrm{OC} \\
\text { stretching }\end{array}$ \\
\hline $1336-1373$ & $1-328$ & $1331-1366$ & $13-1022$ & $\mathrm{CH}$ bending \\
\hline $1376-1406$ & $10-287$ & $1367-1402$ & $17-303$ & $\begin{array}{l}\mathrm{CH} \text { bending }+\mathrm{CH}_{3} \\
\text { scissoring }\end{array}$ \\
\hline $1417-1427$ & $2-53$ & $1409-1421$ & $3-65$ & $\mathrm{CH}_{3}$ scissoring \\
\hline 1447 & 19 & 1434 & 104 & $\mathrm{CH}_{3}$ twisting $(\mathrm{COOH}$ end $)$ \\
\hline 1448 & 159 & 1439 & 148 & $\mathrm{CH}_{3}$ twisting $(\mathrm{OH}$ end) \\
\hline $1494-1532$ & $1-19$ & $1483-1516$ & $3-22$ & $\mathrm{CH}_{3}$ twisting \\
\hline $1876-1909$ & $30-947$ & 1854-1885 & $72-1580$ & $\mathrm{C}=\mathrm{O}$ stretching \\
\hline $3074-3094$ & $7-10$ & $3078-3089$ & $9-18$ & $\mathrm{CH}_{3}$ sym stretching \\
\hline $3110-3153$ & $1-9$ & $3140-3152$ & $2-15$ & $\mathrm{CH}$ stretching \\
\hline $3162-3196$ & $1-16$ & $3164-3196$ & $1-25$ & $\mathrm{CH}_{3}$ asym stretching \\
\hline 3839 & 61 & 3817 & 155 & $\mathrm{OH}$ (free) stretching \\
\hline 3846 & 94 & 3820 & 96 & $\mathrm{OH}(\mathrm{COOH})$ stretching \\
\hline
\end{tabular}

The intensities of the bands appearing in the IR spectra due to $\mathrm{CH}$ bending are much smaller in all five structures than the corresponding bands of PLLA. The band caused by $\mathrm{CH}$ bending and $\mathrm{CH}_{3}$ scissoring vibrations have the same intensity, with the exception the $\beta$-sheet.

The values of the $\mathrm{CH}_{3}$ scissoring vibrations show tremendous growth relative to PLLA. In the $\mathrm{C}=\mathrm{O}$ stretching region the bands are very strong, but they are slightly smaller than the corresponding values in PLLA. The 
solvation increases importantly the intensities of $\mathrm{CH}$ bending and $\mathrm{C}-\mathrm{OC}$ stretching and $\mathrm{C}=\mathrm{O}$ stretching vibrations. In the $1815-1935 \mathrm{~cm}^{-1}$ region are the $\mathrm{C}=\mathrm{O}$ stretching bands of PDLLA. The greatest intensity is shown by the $\beta$-sheet calculated in the solvated model.

Table 2. Peak band assignments for $3_{10}-\mathrm{L}-\mathrm{LA} 10$

\begin{tabular}{|c|c|c|c|c|}
\hline \multicolumn{5}{|c|}{$3{ }_{10}-\mathrm{L}-\mathrm{LA} \mathrm{A}_{10}$} \\
\hline \multicolumn{2}{|c|}{ M062X 6-31G** } & \multicolumn{2}{|c|}{ M062X 6-31G** water } & \multirow[b]{2}{*}{ Assignment } \\
\hline $\mathrm{v}\left(\mathrm{cm}^{-1}\right)$ & Intensity & $\mathrm{v}\left(\mathrm{cm}^{-1}\right)$ & Intensity & \\
\hline $19-226$ & $0-11$ & $17-214$ & $0-8$ & skeletal torsion \\
\hline $234-281$ & $0-14$ & $219-286$ & $0-9$ & $\mathrm{CH}_{3}$ rocking \\
\hline $299-400$ & $0-58$ & $295-396$ & $0-170$ & $\mathrm{CH}_{3}$ wagging \\
\hline 409 & 22 & 399 & 141 & $\mathrm{OH}$ (free) bending \\
\hline $410-416$ & $9-40$ & $403-406$ & $4-8$ & $\mathrm{CH}_{3}$ wagging \\
\hline 419 & 4 & 407 & 5 & $\mathrm{OH}$ (free) bending \\
\hline $425-588$ & $1-230$ & $410-596$ & $1-135$ & CCO bending \\
\hline 636 & 70 & 644 & 63 & $\mathrm{OH}(\mathrm{COOH})$ bending \\
\hline $655-788$ & $0-35$ & $660-775$ & $0-50$ & $\mathrm{C}=\mathrm{O}$ bending \\
\hline 824 & 14 & 842 & 21 & $\begin{array}{l}\text { C-CO (carboxyl) } \\
\text { stretching }\end{array}$ \\
\hline $878-910$ & $1-45$ & 891-909 & $1-37$ & $\begin{array}{l}\mathrm{CH}_{3} \text { bending }+\mathrm{COC} \\
\text { bending }\end{array}$ \\
\hline $925-1106$ & $1-93$ & $925-1099$ & $0-217$ & $\mathrm{CH}_{3}$ rocking \\
\hline $1127-1164$ & $15-106$ & $1104-1161$ & $41-232$ & $\mathrm{C}-\mathrm{CH}_{3}$ stretching \\
\hline $1167-1189$ & $5-146$ & $1162-1185$ & $3-213$ & C-OC stretching \\
\hline $1222-1331$ & $3-89$ & $1213-1329$ & $8-369$ & $\begin{array}{l}\mathrm{CH} \text { bending }+\mathrm{C}-\mathrm{OC} \\
\text { stretching }\end{array}$ \\
\hline $1332-1372$ & $11-403$ & $1331-1365$ & $4-938$ & $\mathrm{CH}$ bending \\
\hline $1373-1403$ & $6-182$ & $1367-1402$ & $11-311$ & $\begin{array}{l}\mathrm{CH} \text { bending }+\mathrm{CH}_{3} \\
\text { scissoring }\end{array}$ \\
\hline $1412-1426$ & $3-88$ & $1407-1421$ & $12-80$ & $\mathrm{CH}_{3}$ scissoring \\
\hline 1445 & 28 & 1430 & 1430 & $\begin{array}{l}\mathrm{CH}_{3} \text { twisting }(\mathrm{COOH} \\
\text { end) }\end{array}$ \\
\hline 1448 & 83 & 1445 & 120 & $\mathrm{CH}_{3}$ twisting $(\mathrm{OH}$ end) \\
\hline $1495-1528$ & $0-46$ & $1486-1518$ & $1-33$ & $\mathrm{CH}_{3}$ twisting \\
\hline $1875-1931$ & $32-756$ & 1852-1894 & $57-1074$ & $\mathrm{C}=\mathrm{O}$ stretching \\
\hline 3078-3094 & $7-10$ & $3071-3091$ & $9-17$ & $\mathrm{CH}_{3}$ sym stretching \\
\hline $3115-3152$ & $1-9$ & $3120-3154$ & $4-13$ & $\mathrm{CH}$ stretching \\
\hline $3171-3193$ & $0-14$ & 3159-3194 & $2-25$ & $\mathrm{CH}_{3}$ asym stretching \\
\hline 3826 & 62 & 3850 & 75 & $\mathrm{OH}$ (free) stretching \\
\hline 3830 & 100 & 3807 & 159 & $\mathrm{OH}(\mathrm{COOH})$ stretching \\
\hline
\end{tabular}


Table 3. Peak band assignments for $\pi-L-L A_{10}$

\begin{tabular}{|c|c|c|c|c|}
\hline \multicolumn{5}{|c|}{$\pi-L-L A_{10}$} \\
\hline \multicolumn{2}{|c|}{ M062X 6-31G** } & \multicolumn{2}{|c|}{ M062X 6-31G** water } & \multirow[b]{2}{*}{ Assignment } \\
\hline $\mathrm{v}\left(\mathrm{cm}^{-1}\right)$ & Intensity & $\mathrm{v}\left(\mathrm{cm}^{-1}\right)$ & Intensity & \\
\hline $18-225$ & $0-6$ & $10-222$ & $0-6$ & skeletal torsion \\
\hline $239-286$ & $0-66$ & $229-291$ & $1-12$ & $\mathrm{CH}_{3}$ rocking \\
\hline $290-398$ & $0-66$ & $296-393$ & $1-157$ & $\mathrm{CH}_{3}$ wagging \\
\hline 403 & 22 & 398 & 47 & $\mathrm{OH}$ (free) bending \\
\hline $408-411$ & $7-26$ & $400-403$ & $3-81$ & $\mathrm{CH}_{3}$ wagging \\
\hline 414 & 6 & 406 & 23 & $\mathrm{OH}$ (free) bending \\
\hline $420-587$ & $2-34$ & $410-586$ & $1-87$ & CCO bending \\
\hline 611 & 73 & 606 & 95 & $\mathrm{OH}(\mathrm{COOH})$ bending \\
\hline $640-790$ & $1-71$ & $638-789$ & $2-78$ & $\mathrm{C}=\mathrm{O}$ bending \\
\hline 832 & 5 & 835 & 8 & $\begin{array}{l}\text { C-CO (carboxyl) } \\
\text { stretching }\end{array}$ \\
\hline $894-915$ & $0-47$ & $890-911$ & $12-70$ & $\begin{array}{l}\mathrm{CH}_{3} \text { bending }+\mathrm{COC} \\
\text { bending }\end{array}$ \\
\hline $928-1102$ & $0-91$ & $927-1097$ & $2-240$ & $\mathrm{CH}_{3}$ rocking \\
\hline $1124-1165$ & $8-85$ & $1106-1157$ & $45-233$ & $\mathrm{C}-\mathrm{CH}_{3}$ stretching \\
\hline $1166-1217$ & $9-150$ & $1163-1186$ & $8-127$ & C-OC stretching \\
\hline $1231-1330$ & $11-192$ & $1216-1334$ & $22-320$ & $\begin{array}{l}\mathrm{CH} \text { bending + } \mathrm{C}-\mathrm{OC} \\
\text { stretching }\end{array}$ \\
\hline 1334-1373 & $6-416$ & $1335-1365$ & $21-586$ & $\mathrm{CH}$ bending \\
\hline $1378-1411$ & $6-222$ & $1368-1407$ & $6-291$ & $\begin{array}{l}\mathrm{CH} \text { bending }+\mathrm{CH}_{3} \\
\text { scissoring }\end{array}$ \\
\hline $1417-1433$ & $6-104$ & $1409-1426$ & $9-104$ & $\mathrm{CH}_{3}$ scissoring \\
\hline 1436 & 53 & 1431 & 110 & $\begin{array}{l}\mathrm{CH}_{3} \text { twisting }(\mathrm{COOH} \\
\text { end) }\end{array}$ \\
\hline 1451 & 3 & 1432 & 121 & $\mathrm{CH}_{3}$ twisting $(\mathrm{OH}$ end) \\
\hline $1494-1530$ & $2-20$ & $1487-1513$ & $0-29$ & $\mathrm{CH}_{3}$ twisting \\
\hline $1865-1910$ & 34-999 & $1854-1878$ & $56-1313$ & $\mathrm{C}=\mathrm{O}$ stretching \\
\hline $3050-3090$ & $6-32$ & $3080-3098$ & $9-21$ & $\mathrm{CH}_{3}$ sym stretching \\
\hline $3093-3154$ & $0-11$ & $3100-3151$ & $2-15$ & $\mathrm{CH}$ stretching \\
\hline $3170-3191$ & $1-12$ & $3166-3201$ & $1-24$ & $\mathrm{CH}_{3}$ asym stretching \\
\hline 3835 & 53 & 3854 & 72 & $\mathrm{OH}$ (free) stretching \\
\hline 3846 & 98 & 3821 & 155 & $\mathrm{OH}(\mathrm{COOH})$ stretching \\
\hline
\end{tabular}

$\mathrm{CH} 3$ scissorings cause bands in $1409-1438 \mathrm{~cm}^{-1}$ region. The intensities are medium, with the solvated $\beta$-sheet showing larger values.

The calculated frequencies $\left(\mathrm{cm}^{-1}\right)$ and peak band assignment for the helical structures and $\beta$-sheet of PDLLA are shown in tables 5-8. 
COMPUTATIONAL INVESTIGATION OF SPECTROSCOPIC PARAMETERS IN PUTATIVE SECONDARY ...

Table 4. Peak band assignments for $\beta-L-L A_{10}$

\begin{tabular}{|c|c|c|c|c|}
\hline \multicolumn{5}{|c|}{$\beta-L-L A_{10}$} \\
\hline \multicolumn{2}{|c|}{ M062X 6-31G** } & \multicolumn{2}{|c|}{ M062X 6-31G** water } & \\
\hline $\mathrm{v}\left(\mathrm{cm}^{-1}\right)$ & Intensity & $\mathrm{v}\left(\mathrm{cm}^{-1}\right)$ & Intensity & Assignment \\
\hline $5-202$ & $0-31$ & $5-203$ & $0-36$ & skeletal torsion \\
\hline $220-313$ & $0-18$ & $208-301$ & $0-145$ & $\mathrm{CH}_{3}$ rocking \\
\hline 317 & 65 & 314 & 8 & $\mathrm{OH}$ (free) bending \\
\hline $327-402$ & $0-18$ & $329-397$ & $1-26$ & $\mathrm{CH}_{3}$ wagging \\
\hline $421-579$ & $0-38$ & $416-578$ & $0-62$ & CCO bending \\
\hline 587 & 79 & 593 & 102 & $\mathrm{OH}(\mathrm{COOH})$ bending \\
\hline $602-801$ & $0-40$ & $601-799$ & $2-134$ & $\mathrm{C}=\mathrm{O}$ bending \\
\hline 844 & 13 & 844 & 14 & C-CO (carboxyl) stretching \\
\hline $896-912$ & $1-22$ & 894-909 & $2-36$ & $\mathrm{CH}_{3}+\mathrm{COC}$ bending \\
\hline $932-1110$ & $0-54$ & $928-1106$ & $0-49$ & $\mathrm{CH}_{3}$ rocking \\
\hline $1130-1145$ & $1-20$ & $1124-1143$ & $0-47$ & $\mathrm{C}-\mathrm{CH}_{3}$ stretching \\
\hline $1157-1213$ & $23-970$ & $1151-1187$ & $3-2171$ & C-OC stretching \\
\hline $1215-1290$ & 4-3164 & $1213-1286$ & 3-3093 & $\mathrm{CH}$ bending + C-OC stretching \\
\hline $1318-1370$ & $1-378$ & 1316-1366 & $1-341$ & $\mathrm{CH}$ bending \\
\hline $1375-1412$ & $1-83$ & $1369-1407$ & $2-54$ & $\mathrm{CH}$ bending $+\mathrm{CH}_{3}$ scissoring \\
\hline 1414-1437 & 4-332 & $1408-1433$ & $8-455$ & $\mathrm{CH}_{3}$ scissoring \\
\hline 1447 & 76 & 1444 & 109 & $\mathrm{CH}_{3}$ twisting $(\mathrm{COOH}$ end $)$ \\
\hline 1468 & 5 & 1459 & 9 & $\mathrm{CH}_{3}$ twisting $(\mathrm{OH}$ end $)$ \\
\hline $1508-1518$ & $5-55$ & 1495-1509 & $2-75$ & $\mathrm{CH}_{3}$ twisting \\
\hline 1865-1932 & $9-563$ & $1850-1898$ & 4-1293 & $\mathrm{C}=\mathrm{O}$ stretching \\
\hline $3058-3095$ & $5-23$ & $3076-3104$ & $8-22$ & $\mathrm{CH}_{3}$ sym stretching \\
\hline $3117-3130$ & $5-22$ & $3125-3134$ & $8-16$ & $\mathrm{CH}$ stretching \\
\hline $3169-3196$ & $3-14$ & 3163-3196 & $7-25$ & $\mathrm{CH}_{3}$ asym stretching \\
\hline 3829 & 112 & 3819 & 103 & $\mathrm{OH}$ (free) stretching \\
\hline 3810 & 81 & 3806 & 169 & $\mathrm{OH}(\mathrm{COOH})$ stretching \\
\hline
\end{tabular}

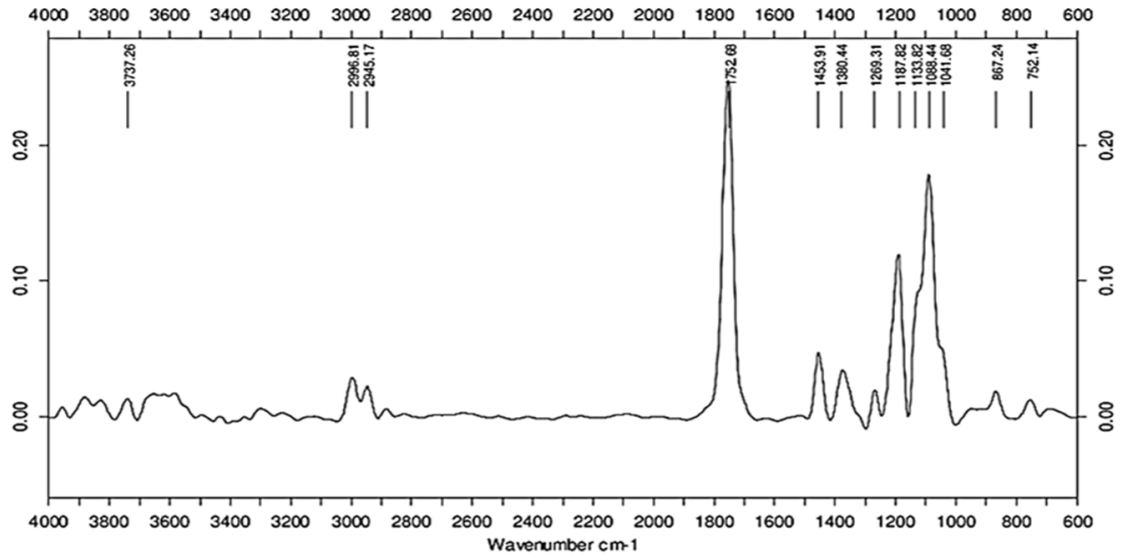

Figure 4. IR spectrum of poly(DL-lactic acid). 

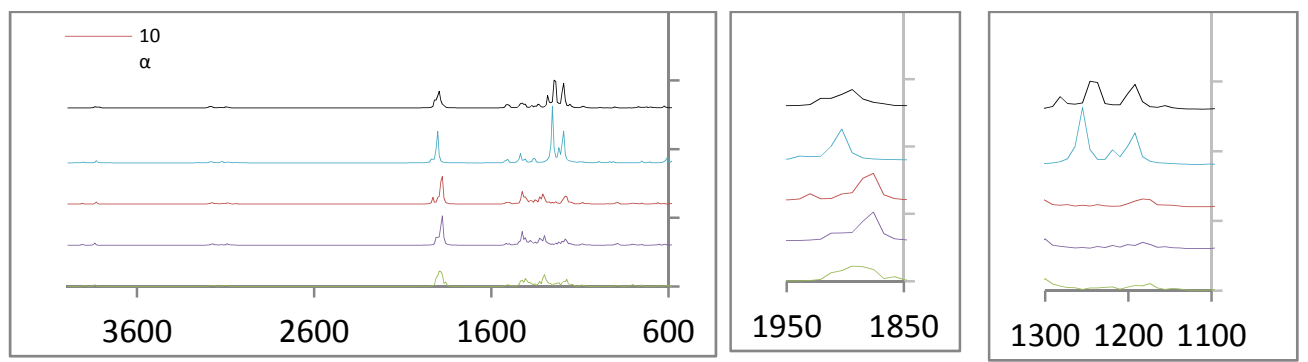

Figure 5. IR spectra of PDLLA (M062x/6-31G**):(a) full spectrum; (b) carbonyl stretching region; (c) backbone stretching region

Table 5. Peak band assignments for $\alpha-D L-L A_{10}$

\begin{tabular}{|c|c|c|c|c|}
\hline \multicolumn{5}{|c|}{$\alpha-D L-L A_{10}$} \\
\hline \multicolumn{2}{|c|}{ M062X 6-31G** } & \multicolumn{2}{|c|}{ M062X 6-31G** water } & \multirow[b]{2}{*}{ Assignment } \\
\hline $\mathrm{v}\left(\mathrm{cm}^{-1}\right)$ & Intensity & $\mathrm{v}\left(\mathrm{cm}^{-1}\right)$ & Intensity & \\
\hline $8-190$ & $0-6$ & $13-189$ & $0-9$ & Skeletal torsion \\
\hline $199-241$ & $0-44$ & $201-237$ & $0-5$ & $\mathrm{CH}_{3}$ rocking \\
\hline 242 & 56 & 238 & $1-5$ & $\mathrm{OH}$ (free) bending \\
\hline $246-290$ & $1-7$ & $241-294$ & $0-18$ & $\mathrm{CH}_{3}$ rocking \\
\hline $302-408$ & $0-26$ & $298-422$ & $1-156$ & $\mathrm{CH}_{3}$ wagging \\
\hline $428-580$ & $1-47$ & 427-567 & $2-96$ & CCO bending \\
\hline 585 & 41 & 576 & 63 & $\mathrm{OH}(\mathrm{COOH})$ bending \\
\hline 593-807 & $0-57$ & $603-802$ & $1-87$ & $\mathrm{C}=\mathrm{O}$ bending \\
\hline 819 & 22 & 821 & 37 & C-CO (carboxyl) stretching \\
\hline $881-900$ & $3-56$ & $881-900$ & $2-63$ & $\mathrm{CH}_{3}$ bending $+\mathrm{COC}$ bending \\
\hline $923-1092$ & $1-35$ & $923-1087$ & $1-71$ & $\mathrm{CH}_{3}$ rocking \\
\hline $1139-1168$ & $3-39$ & $1130-1164$ & $10-90$ & $\mathrm{C}-\mathrm{CH}_{3}$ stretching \\
\hline $1175-1199$ & $12-296$ & $1167-1198$ & $17-289$ & C-OC stretching \\
\hline $1232-1323$ & $10-320$ & $1225-1324$ & $22-838$ & $\mathrm{CH}$ bending $+\mathrm{C}-\mathrm{OC}$ stretching \\
\hline $1327-1380$ & $7-163$ & $1326-1374$ & $17-258$ & $\mathrm{CH}$ bending \\
\hline $1383-1416$ & $13-147$ & $1378-1412$ & $7-294$ & $\mathrm{CH}$ bending $+\mathrm{CH}_{3}$ scissoring \\
\hline $1419-1429$ & $7-287$ & $1414-1424$ & $14-230$ & $\mathrm{CH}_{3}$ scissoring \\
\hline 1436 & 29 & 1437 & 62 & $\mathrm{CH}_{3}$ twisting $(\mathrm{COOH}$ end $)$ \\
\hline 1464 & 31 & 1451 & 40 & $\mathrm{CH}_{3}$ twisting $(\mathrm{OH}$ end) \\
\hline $1492-1528$ & $0-35$ & $1481-1515$ & $1-30$ & $\mathrm{CH}_{3}$ twisting \\
\hline $1871-1930$ & $42-875$ & $1854-1888$ & $127-1047$ & $\mathrm{C}=\mathrm{O}$ stretching \\
\hline 3039 & 28 & 3029 & 40 & $\mathrm{CH}$ stretching \\
\hline $3073-3101$ & 3-17 & $3082-3102$ & $4-20$ & $\mathrm{CH}_{3}$ sym stretching \\
\hline $3111-3137$ & $1-13$ & $3115-3150$ & $5-11$ & $\mathrm{CH}$ stretching \\
\hline $3153-3213$ & $1-26$ & $3167-3209$ & $2-25$ & $\mathrm{CH}_{3}$ asym stretching \\
\hline 3907 & 48 & 3882 & 78 & $\mathrm{OH}$ (free) stretching \\
\hline 3830 & 103 & 3809 & 160 & $\mathrm{OH}(\mathrm{COOH})$ stretching \\
\hline
\end{tabular}


COMPUTATIONAL INVESTIGATION OF SPECTROSCOPIC PARAMETERS IN PUTATIVE SECONDARY ...

Table 6. Peak band assignments for $10_{3}-D L-L A_{10}$

\begin{tabular}{|c|c|c|c|c|}
\hline \multicolumn{5}{|c|}{$10_{3}-\mathrm{DL}-\mathrm{LA}_{10}$} \\
\hline M062X & $31 G^{* *}$ & M062X 6-3 & ${ }^{* *}$ water & \\
\hline $\mathrm{v}\left(\mathrm{cm}^{-1}\right)$ & Intensity & $\mathrm{v}\left(\mathrm{cm}^{-1}\right)$ & Intensity & Assignment \\
\hline $15-203$ & $0-6$ & $23-184$ & $0-8$ & Skeletal torsion \\
\hline 211-248 & $0-73$ & $191-227$ & $0-3$ & $\mathrm{CH}_{3}$ rocking \\
\hline 249 & $0-6$ & $23-184$ & $0-8$ & $\mathrm{OH}$ (free) bending \\
\hline $252-292$ & $1-5$ & $236-291$ & $1-10$ & $\mathrm{CH}_{3}$ rocking \\
\hline $295-420$ & $0-17$ & $298-420$ & $2-192$ & $\mathrm{CH}_{3}$ wagging \\
\hline $428-572$ & $2-39$ & $431-560$ & 3-105 & CCO bending \\
\hline 581 & 10 & 564 & 28 & $\mathrm{OH}(\mathrm{COOH})$ bending \\
\hline $604-808$ & $0-76$ & $601-798$ & $0-141$ & $\mathrm{C}=\mathrm{O}$ bending \\
\hline 826 & 10 & 814 & 21 & C-CO (carboxyl) stretching \\
\hline $882-903$ & $2-43$ & $867-889$ & $1-135$ & $\begin{array}{l}\mathrm{CH}_{3} \text { bending }+\mathrm{COC} \\
\text { bending }\end{array}$ \\
\hline 924-1093 & $1-28$ & $911-1080$ & $1-123$ & $\mathrm{CH}_{3}$ rocking \\
\hline $1138-1171$ & $1-40$ & $1111-1141$ & $31-258$ & $\mathrm{C}-\mathrm{CH}_{3}$ stretching \\
\hline 1174-1202 & $13-120$ & $1146-1177$ & $10-264$ & C-OC stretching \\
\hline $1218-1325$ & $28-455$ & $1204-1313$ & $62-1119$ & $\begin{array}{l}\mathrm{CH} \text { bending + C-OC } \\
\text { stretching }\end{array}$ \\
\hline $1330-1384$ & $7-203$ & $1316-1368$ & $11-286$ & $\mathrm{CH}$ bending \\
\hline $1385-1419$ & $11-139$ & 1369-1407 & $4-310$ & $\begin{array}{l}\mathrm{CH} \text { bending }+\mathrm{CH}_{3} \\
\text { scissoring }\end{array}$ \\
\hline $1421-1429$ & $7-248$ & $1410-1418$ & $13-40$ & $\mathrm{CH}_{3}$ scissoring \\
\hline 1443 & 115 & 1428 & 66 & $\mathrm{CH}_{3}$ twisting $(\mathrm{COOH}$ end $)$ \\
\hline 1464 & 37 & 1444 & 28 & $\mathrm{CH}_{3}$ twisting $(\mathrm{OH}$ end $)$ \\
\hline $1496-1533$ & $0-26$ & $1486-1513$ & $6-20$ & $\mathrm{CH}_{3}$ twisting \\
\hline $1871-1910$ & $62-672$ & $1815-1840$ & $31-1034$ & $\mathrm{C}=\mathrm{O}$ stretching \\
\hline 3052 & 26 & 3045 & 34 & $\mathrm{CH}$ stretching \\
\hline $3073-3096$ & $3-21$ & $3071-3097$ & $8-15$ & $\mathrm{CH}_{3}$ sym stretching \\
\hline $3108-3141$ & $2-8$ & $3117-3155$ & $2-22$ & $\mathrm{CH}$ stretching \\
\hline $3154-3215$ & $0-25$ & $3158-3196$ & $1-22$ & $\mathrm{CH}_{3}$ asym stretching \\
\hline 3839 & 104 & 3764 & 159 & $\mathrm{OH}(\mathrm{COOH})$ stretching \\
\hline 3907 & 50 & 3854 & 76 & $\mathrm{OH}$ (free) stretching \\
\hline
\end{tabular}

Comparing the calculated data with the experimental data it can be seen that the calculated frequency values are bigger than the experimental values. 
IZABELLA IRSAI, ALEXANDRU LUPAN, CORNELIA MAJDIK, RADU SILAGHI-DUMITRESCU

Table 7. Peak band assignments for $\pi-D L-L A_{10}$

\begin{tabular}{|c|c|c|c|c|}
\hline \multicolumn{5}{|c|}{$\pi-D L-L A_{10}$} \\
\hline \multicolumn{2}{|c|}{ M062X 6-31G** } & \multicolumn{2}{|c|}{ M062X 6-31G** water } & \multirow[b]{2}{*}{ Assignment } \\
\hline $\mathrm{v}\left(\mathrm{cm}^{-1}\right)$ & Intensity & $\mathrm{v}\left(\mathrm{cm}^{-1}\right)$ & Intensity & \\
\hline $15-200$ & $0-5$ & $14-186$ & $0-10$ & Skeletal torsion \\
\hline $202-247$ & $0-3$ & $192-236$ & $0-7$ & $\mathrm{CH}_{3}$ rocking \\
\hline 249 & 3 & 240 & 4 & $\mathrm{OH}$ (free) bending \\
\hline $253-290$ & $1-53$ & $242-296$ & $0-21$ & $\mathrm{CH}_{3}$ rocking \\
\hline $304-405$ & $1-26$ & $298-409$ & $1-97$ & $\mathrm{CH}_{3}$ wagging \\
\hline $437-565$ & $3-36$ & $410-568$ & $2-59$ & CCO bending \\
\hline 585 & 11 & 572 & 15 & $\mathrm{OH}(\mathrm{COOH})$ bending \\
\hline $588-805$ & $1-72$ & $596-803$ & $1-141$ & $\mathrm{C}=\mathrm{O}$ bending \\
\hline 832 & 9 & 825 & 48 & C-CO stretching \\
\hline $879-903$ & $2-25$ & $857-902$ & $0-43$ & $\begin{array}{l}\mathrm{CH}_{3} \text { bending }+\mathrm{COC} \\
\text { bending }\end{array}$ \\
\hline 923-1099 & $1-32$ & 923-1087 & $1-96$ & $\mathrm{CH}_{3}$ rocking \\
\hline 1134-1169 & $4-59$ & $1125-1163$ & $11-144$ & $\mathrm{C}-\mathrm{CH}_{3}$ stretching \\
\hline 1171-1199 & $31-144$ & $1167-1196$ & $36-185$ & C-OC stretching \\
\hline $1221-1323$ & $20-433$ & $1209-1323$ & $24-1048$ & $\begin{array}{l}\mathrm{CH} \text { bending }+\mathrm{C}-\mathrm{OC} \\
\text { stretching }\end{array}$ \\
\hline $1329-1379$ & $6-177$ & $1331-1372$ & $11-213$ & $\mathrm{CH}$ bending \\
\hline $1386-1417$ & $14-209$ & $1377-1412$ & $12-178$ & $\begin{array}{l}\mathrm{CH} \text { bending }+\mathrm{CH}_{3} \\
\text { scissoring }\end{array}$ \\
\hline $1421-1438$ & $1161-101$ & 1413-1429 & $6-236$ & $\mathrm{CH}_{3}$ scissoring \\
\hline 1440 & 40 & 1437 & 106 & $\mathrm{CH}_{3}$ twisting $(\mathrm{COOH}$ end $)$ \\
\hline 1466 & 18 & 1454 & 33 & $\mathrm{CH}_{3}$ twisting $(\mathrm{OH}$ end) \\
\hline $1488-1526$ & $3-20$ & $1482-1516$ & $2-32$ & $\mathrm{CH}_{3}$ twisting \\
\hline $1856-1910$ & $29-531$ & $1854-1881$ & $87-886$ & $\mathrm{C}=\mathrm{O}$ stretching \\
\hline 3066 & 12 & 3031 & 40 & $\mathrm{CH}$ stretching \\
\hline $3084-3102$ & $3-15$ & $3081-3101$ & $4-19$ & $\mathrm{CH}_{3}$ sym stretching \\
\hline $3109-3154$ & $2-9$ & $3118-3153$ & $5-14$ & $\mathrm{CH}$ stretching \\
\hline $3163-3210$ & $1-29$ & $3167-3208$ & $2-22$ & $\mathrm{CH}_{3}$ asym stretching \\
\hline 3893 & 51 & 3882 & 79 & $\mathrm{OH}$ (free) stretching \\
\hline 3836 & 102 & 3815 & 156 & $\mathrm{OH}(\mathrm{COOH})$ stretching \\
\hline
\end{tabular}


COMPUTATIONAL INVESTIGATION OF SPECTROSCOPIC PARAMETERS IN PUTATIVE SECONDARY ...

Table 8. Peak band assignments for $\beta-D L-L A_{10}$

\begin{tabular}{|c|c|c|c|c|}
\hline \multicolumn{5}{|c|}{$\beta-D L-\mathrm{LA}_{10}$} \\
\hline \multicolumn{2}{|c|}{ M062X 6-31G** } & \multicolumn{2}{|c|}{ M062X 6-31G** water } & \\
\hline $\mathrm{v}\left(\mathrm{cm}^{-1}\right)$ & Intensity & $\mathrm{v}\left(\mathrm{cm}^{-1}\right)$ & Intensity & Assignment \\
\hline 4-197 & $0-47$ & $5-194$ & $0-41$ & Skeletal torsion \\
\hline $212-244$ & $0-7$ & $208-240$ & $0-4$ & $\mathrm{CH}_{3}$ rocking \\
\hline 248 & 23 & 244 & 2 & $\mathrm{OH}$ (free) bending \\
\hline $256-318$ & $1-11$ & $249-316$ & $0-161$ & $\mathrm{CH}_{3}$ rocking \\
\hline $329-389$ & $0-16$ & $320-384$ & $1-22$ & $\mathrm{CH}_{3}$ wagging \\
\hline $396-600$ & $0-71$ & $390-602$ & $0-102$ & CCO bending \\
\hline 607 & 205 & 615 & 24 & $\mathrm{OH}(\mathrm{COOH})$ bending \\
\hline $613-808$ & $1-45$ & $616-803$ & $1-224$ & $\mathrm{C}=\mathrm{O}$ bending \\
\hline 844 & 11 & 846 & 14 & C-CO (carboxyl) stretching \\
\hline $896-912$ & $2-43$ & $891-908$ & $1-29$ & $\begin{array}{l}\mathrm{CH}_{3} \text { bending + } \mathrm{COC} \\
\text { bending }\end{array}$ \\
\hline $931-1105$ & $1-46$ & 926-1104 & $1-93$ & $\mathrm{CH}_{3}$ rocking \\
\hline 1126-1146 & $1-24$ & $1121-1140$ & $2-32$ & $\mathrm{C}-\mathrm{CH}_{3}$ stretching \\
\hline $1152-1215$ & $9-1571$ & $1147-1201$ & $5-1311$ & C-OC stretching \\
\hline $1217-1286$ & $1-2570$ & $1211-1283$ & $3-2270$ & $\begin{array}{l}\mathrm{CH} \text { bending }+\mathrm{C}-\mathrm{OC} \\
\text { stretching }\end{array}$ \\
\hline $1295-1367$ & $7-166$ & 1301-1363 & $13-150$ & $\mathrm{CH}$ bending \\
\hline $1367-1411$ & $1-102$ & $1365-1406$ & $0-80$ & $\begin{array}{l}\mathrm{CH} \text { bending }+\mathrm{CH}_{3} \\
\text { scissoring }\end{array}$ \\
\hline $1412-1435$ & $1-254$ & 1409-1435 & $1-403$ & $\mathrm{CH}_{3}$ scissoring \\
\hline 1445 & 88 & 1449 & 102 & $\mathrm{CH}_{3}$ twisting $(\mathrm{COOH}$ end $)$ \\
\hline 1454 & 37 & 1452 & 55 & $\mathrm{CH}_{3}$ twisting $(\mathrm{OH}$ end $)$ \\
\hline $1507-1523$ & $3-37$ & $1495-1516$ & $6-57$ & $\mathrm{CH}_{3}$ twisting \\
\hline 1900-1935 & $24-888$ & 1870-1901 & $5-2423$ & $\mathrm{C}=\mathrm{O}$ stretching \\
\hline 3042 & 28 & 3065 & 34 & $\mathrm{CH}$ stretching \\
\hline $3075-3092$ & $5-15$ & $3077-3094$ & $8-321$ & $\mathrm{CH}_{3}$ sym stretching \\
\hline $3114-3125$ & $9-13$ & $3128-3137$ & $5-27$ & $\mathrm{CH}$ stretching \\
\hline $3156-3198$ & $0-20$ & $3162-3199$ & $7-23$ & $\mathrm{CH}_{3}$ asym stretching \\
\hline 3827 & 110 & 3797 & 170 & $\mathrm{OH}(\mathrm{COOH})$ stretching \\
\hline 3903 & 39 & 3887 & 81 & $\mathrm{OH}$ (free) stretching \\
\hline
\end{tabular}

\section{NMR}

Another important tool to characterize the structure of the polymer is the NMR spectroscopy. It was used ${ }^{1} \mathrm{H}$ NMR and ${ }^{13} \mathrm{C}$ NMR spectroscopy. In the ${ }^{1} \mathrm{H}$ NMR spectra the determined chemical shifts correspond to $\mathrm{CH}$ and $\mathrm{CH}_{3}$ resonance. $\mathrm{CO}, \mathrm{CH}_{3}$ and $\mathrm{CH}$ resonance are found in ${ }^{13} \mathrm{C}$ NMR spectra. The ${ }^{1} \mathrm{H}$ NMR spectra of the PDLLA obtained by polycondensation of DL-lactic acid is shown in figure 6 . The NMR analysis of poly(lactic acid) were found 
considerably improved by recording spectra in DMSO- $\mathrm{d}_{6}$ instead of $\mathrm{CDCl}_{3}$ [27].The most intensive signals were those located at 1.45 and 4.93 respectively 1.46 and $5.16 \mathrm{ppm}$ which correspond to $\mathrm{CH}$ and $\mathrm{CH}_{3}$ resonance in PLLA respectively PDLLA.

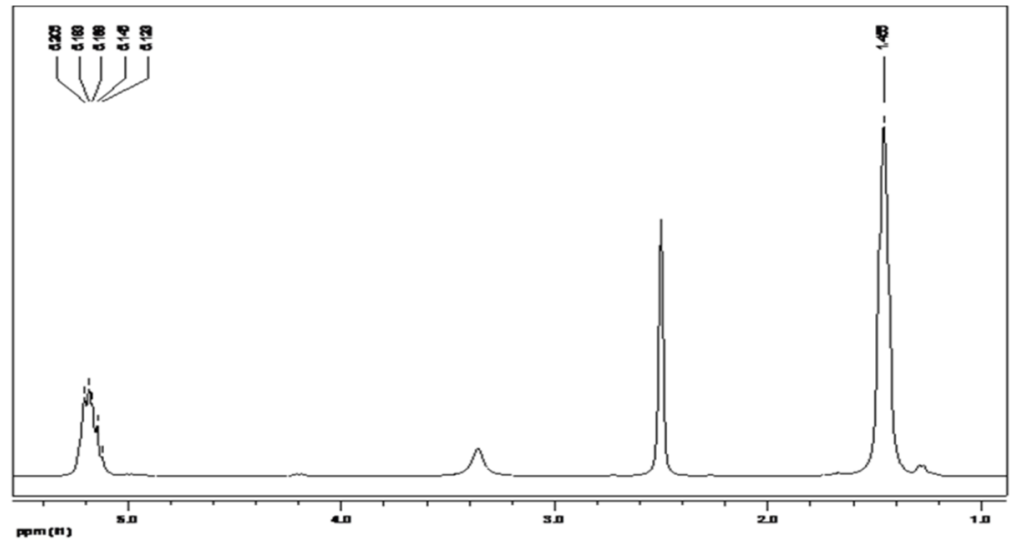

Figure 6. ${ }^{1} \mathrm{H}-\mathrm{NMR}$ spectrum of PDLLA.

The simulated ${ }^{1} \mathrm{H}$ NMR spectrum of PLLA is shown in figure 7.

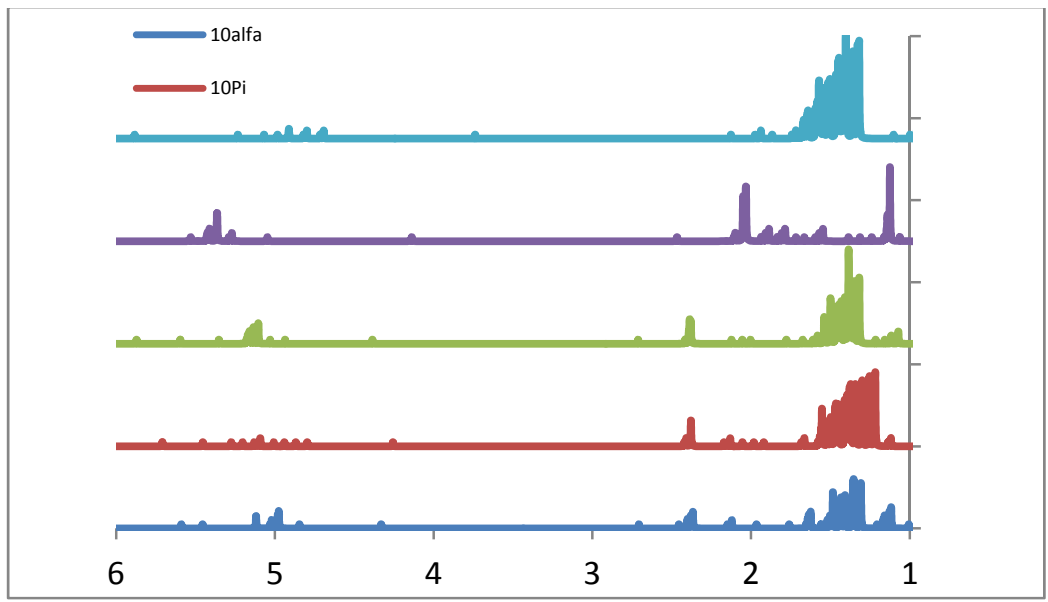

Figure 7. ${ }^{1} \mathrm{H}$ NMR spectra of PLLA (M062x/6-31G**). 
The shifts of ${ }^{13} \mathrm{C}$ NMR spectrum of the four secondary-type structure of PLLA calculated by DFT/M062x/6-31G** method are larger than those calculated in solvated models (Table 9.). The ${ }^{1} \mathrm{H}$ NMR chemical shifts of PLLA are not always smaller in solution (Table 10.). Taking into account all five NMR signals covered by these two Tables, the $\beta$ and $\pi$ structures appear to generally yield the closest values to the experiment.

Table 9. Computed and experimental ${ }^{13} \mathrm{C}$ NMR Chemical shifts ( $\delta$, in ppm) of $\mathrm{CO}, \mathrm{CH}$ and $\mathrm{CH}_{3}$ of PLLA in vacuum (v) and water (w), respectively

\begin{tabular}{|c|c|c|c|c|c|c|c|c|c|}
\hline PLLA & \multicolumn{2}{|c|}{$\alpha$} & \multicolumn{2}{|c|}{$\pi$} & \multicolumn{2}{|c|}{$10_{3}$} & \multicolumn{2}{|c|}{$\beta$} & \multirow{2}{*}{ Exp. } \\
\hline & $\mathrm{v}$ & $\mathrm{w}$ & $\mathrm{v}$ & $\mathrm{w}$ & $\mathrm{v}$ & $\mathrm{w}$ & $\mathrm{v}$ & $\mathrm{w}$ & \\
\hline$\delta(\mathrm{CO})$ & 178 & 175 & 178 & 175 & 177 & 175 & 175 & 172 & 172 \\
\hline$\delta(\mathrm{CH})$ & 72 & 68 & 72 & 68 & 72 & 68 & 72 & 67 & 66 \\
\hline$\delta\left(\mathrm{CH}_{3}\right)$ & 19 & 13 & 19 & 13 & 18 & 13 & 19 & 14 & 17 \\
\hline
\end{tabular}

Table 10. Computed and experimental ${ }^{1} \mathrm{H}$ NMR Chemical shifts $(\delta$, in ppm) of $\mathrm{CH}$ and $\mathrm{CH}_{3}$ of PLLA in vacuum (v) and water (w), respectively

\begin{tabular}{|c|c|c|c|c|c|c|c|c|c|}
\hline PLLA & \multicolumn{2}{|c|}{$\alpha$} & \multicolumn{2}{|c|}{$\Pi$} & \multicolumn{2}{|c|}{$10_{3}$} & \multicolumn{2}{|c|}{$\beta$} & \multirow{2}{*}{ Exp. } \\
\hline & v & w & v & w & v & w & v & w & \\
\hline$\delta(\mathrm{CH})$ & 5.2 & 5.1 & 5.1 & 5.1 & 5.2 & 5.1 & 5.3 & 5.5 & 4.9 \\
\hline$\delta\left(\mathrm{CH}_{3}\right)$ & 1.7 & 1.7 & 1.7 & 1.6 & 1.7 & 1.7 & 1.7 & 1.6 & 1.5 \\
\hline
\end{tabular}

Figure 8 shows the calculated ${ }^{1} \mathrm{H}$ NMR spectrum of PDLLA.

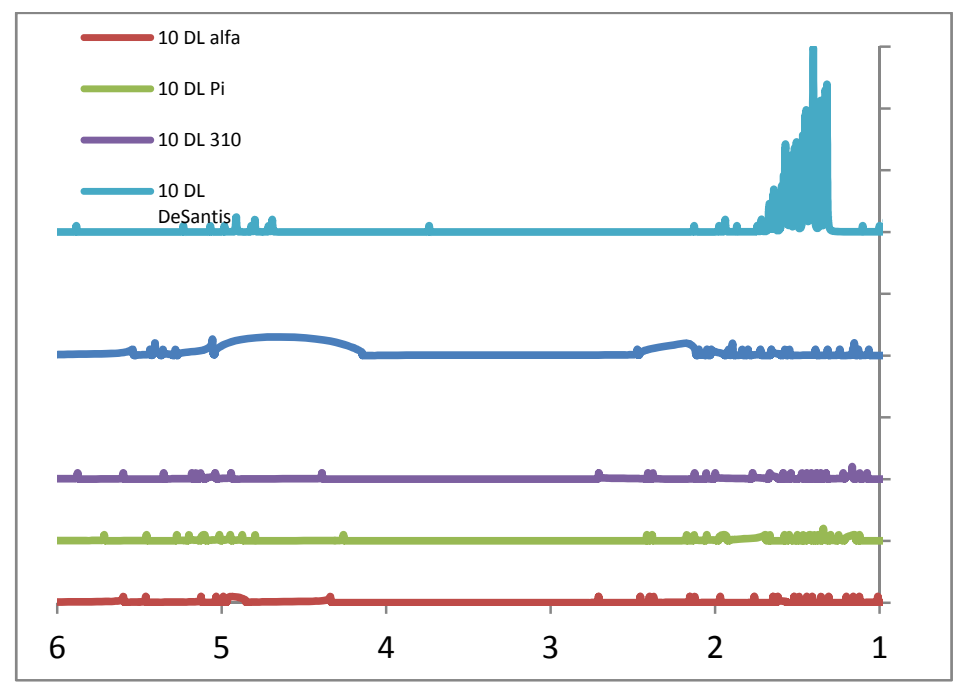

Figure 8. ${ }^{1} \mathrm{H}$ NMR spectra of PDLLA (M062x/6-31G**). 
Same as in the case of PLLA the chemical shifts of ${ }^{13} \mathrm{C}$ NMR spectrum of PDDLA show smaller values in solvated model (Table 11.). The ${ }^{1} \mathrm{H}$ NMR shifts due to $\mathrm{CH}$ resonance are bigger in all four structures (Table 12.).

Table 11. ${ }^{13} \mathrm{C}$ NMR Chemical shifts $\left(\delta\right.$, in ppm) of $\mathrm{CO}, \mathrm{CH}$ and $\mathrm{CH}_{3}$ of PDLLA in vacuum $(v)$ and water $(w)$, respectively

\begin{tabular}{|c|c|c|c|c|c|c|c|c|c|}
\hline PDLLA & \multicolumn{2}{|c|}{$\alpha$} & \multicolumn{2}{|c|}{$\pi$} & \multicolumn{2}{|c|}{$10_{3}$} & \multicolumn{2}{|c|}{$\beta$} & \multirow{2}{*}{ Exp. } \\
\hline & $\mathrm{v}$ & $\mathrm{w}$ & $\mathrm{v}$ & $\mathrm{w}$ & $\mathrm{v}$ & $\mathrm{w}$ & $\mathrm{v}$ & $\mathrm{w}$ & \\
\hline$\delta(\mathrm{CO})$ & 177 & 174 & 177 & 174 & 178 & 176 & 175 & 172 & 169 \\
\hline$\delta(\mathrm{CH})$ & 73 & 68 & 73 & 69 & 73 & 67 & 71 & 67 & 69 \\
\hline$\delta\left(\mathrm{CH}_{3}\right)$ & 18 & 13 & 18 & 13 & 18 & 13 & 19 & 14 & 16 \\
\hline
\end{tabular}

Table 12. ${ }^{1} \mathrm{H}$ NMR Chemical shifts $\left(\delta\right.$, in ppm) of $\mathrm{CH}$ and $\mathrm{CH}_{3}$ of PDLLA in vacuum $(v)$ and water $(w)$, respectively

\begin{tabular}{|c|c|c|c|c|c|c|c|c|c|}
\hline PDLLA & \multicolumn{2}{|c|}{$\alpha$} & \multicolumn{2}{|c|}{$\pi$} & \multicolumn{2}{|c|}{$10_{3}$} & \multicolumn{2}{|c|}{$\beta$} & \multirow{2}{*}{ Exp. } \\
\hline & $\mathrm{v}$ & $\mathrm{w}$ & $\mathrm{v}$ & $\mathrm{w}$ & $\mathrm{v}$ & $\mathrm{w}$ & $\mathrm{v}$ & $\mathrm{w}$ & \\
\hline$\delta(\mathrm{CH})$ & 5.0 & 5.1 & 5.1 & 5.1 & 5.1 & 5.1 & 5.4 & 5.5 & 5.2 \\
\hline$\delta\left(\mathrm{CH}_{3}\right)$ & 1.7 & 1.7 & 1.6 & 1.6 & 1.7 & 1.6 & 1.5 & 1.6 & 1.5 \\
\hline
\end{tabular}

The NMR spectra of PLA can give information about the stereochemistry of the composition. The $\mathrm{CO}$ chemical shifts are larger in the $\alpha, \pi$ and $\beta$ forms of PLLA, while the $\mathrm{CH}_{3}$ shifts are larger in the case of the three helical structures of PLLA. The chemical shifts of ${ }^{1} \mathrm{H}$ NMR spectra of PLLA show larger values in all cases except the $\mathrm{CH}$ shifts of $\beta$-sheet.

\section{CONCLUSIONS}

Geometry optimization performed on polylactic acid at different levels of DFT methods suggest that the most stable of the four structures is the $\pi$ helix and the least stable is the $\beta$ sheet.

The calculated chemical shifts of both ${ }^{13} \mathrm{C}$ NMR and ${ }^{1} \mathrm{H}$ NMR are slightly larger than the experimental one. The solvation reduces the value of the NMR chemical shifts.

The secondary structure of poly(lactic acid) cannot be conclusively clarified from the calculated IR and NMR spectra, suggesting either a need for using more appropriate computational methods or the occurrence of previously unconsidered elements of secondary structure, or the total lack thereof. 


\section{EXPERIMENTAL SECTION}

Materials. Aqueous solutions of L-(+)-lactic acid from Sigma-Aldrich (80\%), DL-lactic acid from Fluka (90\%) were used. PLLA and PDLLA were synthetized by direct dehydropolycondensation. Lactic acid, toluene and $0.1 \%$ tin(II) 2-ethylhexanoate catalyst were mixed into a reaction vessel equipped with a Dean-Stark-type condenser, and heated to the refluxing temperature of the solvent. The reaction time was $20 \mathrm{~h}$. The final product was dissolved in chloroform and precipitated in diethyl ether for purification. The polymer was then filtered out from diethyl ether and dried under vacuum.

Measurements. NMR spectra were recorded with a Bruker Avance 300 spectrometer at the following frequencies: ${ }^{1} \mathrm{H}, 300.13 \mathrm{MHz} ;{ }^{13} \mathrm{C}, 75.47$ $\mathrm{MHz}$ (reference TMS) with DMSO- $\mathrm{d}_{6}$ as the sovent. IR spectra were recorded with a Vector 22 Bruker spectrometer by direct introduction method and a Jasco FT/IR Specord 600 spectometer in $\mathrm{KBr}$ pills. The molecular weights were determined by MALDI-TOF MS (Matrix Assisted Laser Desorption lonization) analysis with a Bruker BIFLEX III ${ }^{\mathrm{TM}}$ spectrometer.

Molecular simulation. A vibrational analysis and NMR simulation has been carried out to analyze the secondary structure of poly(lactic acid) resulted from esterification of ten lactic acid units, hereafter referred to as $\mathrm{LA}_{10}$. These models were built in the Hyperchem [28] software package using built-in options of the Editor module for creating helical structures as well as sheet. Spectroscopic parameters were predicted for helical $(\alpha, \pi, 103)$ and $\beta$ sheet structures, in an attempt to aid our on-going efforts in the synthesis and characterization of poly(lactic acid) variants.

The methods tested here include density functional (M062X/6-31G*, M062X/6-31G**, solvated M062X/6-31G** applied with standard convergence criteria as defined in Gaussian 09 [29]. Spectral parameters were invoked using the commands Freq and NMR. In terms of the importance of solvation, this is estimated by comparing values computed in water (as a limit of very polar medium) and vacuum (as a limit of completely non-polar medium). Further detail on geometry optimizations and on relative energies of the structures are given elsewhere [30].

\section{ACKNOWLEDGMENTS}

This work was supported by CNCSIS-UEFISCDI, projects PNII - ID $312 / 2008$ (RSD and AL), project Parteneriate 72152/2008 (to CM) and by a PhD scholarship from Contract POSDRU/88/1.5/S/60185 - "Innovative doctoral studies in a knowledge based society" (to II). 


\section{REFERENCES}

1. R.A. Jain, Biomaterials, 2000, 21, 2475.

2. A. Dev, N.S. Binulal, A. Anitha, S.V. Nair, T. Furuike, H. Tamura, R. Jayakumar, Carbohydrate Polymers, 2010, 80, 833.

3. M.A. Ibrahim, A. Ismail, M.I. Fetou, A. Gopferich, Journal of Controlled Release, 2005, 106, 241.

4. S. Fredenberg, M. Wahlgren, M. Reslow, A. Axelsson, International Journal of Pharmaceutics, 2011, 415, 34.

5. T. Miyata, T. Masuko, Polymer, 1997, 38, 4003.

6. J. Kobayashi, T. Asahi, M. Ichiki, A. Okikawa, H. Suzuki, T. Watanabe, E. Fukada, Y. Shikinami, Journal of Applied Physics, 1995, 77, 2957.

7. W. Hoogsteen, A.R. Postema, A.J. Pennings, G.G ten Brinke, P. Zugenmaier, Macromolecules, 1990, 23, 634.

8. S. Sasaki, T. Asakura, Macromolecules, 2003, 36, 8385.

9. D. Brizzolara, H.J. Cantow, K. Diederichs, E. Keller, A.J. Domb, Macromolecules, 1996, 29, 191.

10. C. Aleman, B. Lotz, J. Puiggali, Macromolecules, 2001, 34, 4795.

11. P. De Santis, J. Kovacs, Biopolymers, 1968, 6, 299.

12. J. Puiggali, Y. Ikada, H. Tsuji, L. Cartier, T. Okihara, B. Lotz, Polymer, 2000, 41, 8921.

13. T. Okihara, M. Tsuji, A. Kawagushi, K.I. Katayama, H. Tsuji, S.H. Hyon, Y. Ikada, Journal of Macromolecular Science Physics B, 1991, 30, 119.

14. L. Cartier, T. Okihara, Y. Ikada, H. Tsuji, J. Puiggali, B. Lotz, Polymer, 2000, 41, 8909.

15. Y. Ikada, K. Jamshidi, H. Tsuji, S.H. Hyon, Macromolecules, 1987, 20, 904.

16. H. Tsuji, Macromolecular Bioscience, 2005, 5, 569.

17. H. Tsuji; Y. Ikada, Polymer, 1999, 40, 6699.

18. H. Tsuji, I. Fukui, Polymer, 2003, 44, 2891.

19. D. Sawai, Y. Tsugane, M. Tamada, T. Kanamoto, M. Sungil, S.H. Hyon, Journal Polymer Science, Part B: Polymer Physics, 2007, 45, 2632.

20. N. Rahman, T. Kawai, G. Matsuba, K. Nishida, T. Kanaya, H. Watanabe, H. Okamoto, M. Kato, A. Usuki, M. Matsuda, K. Nakajima, N. Honma, Macromolecules, 2009, 42, 4739.

21. S. Kang, S.L. Hsu, H.D. Stidham, B.P. Smith, A. Leugers, X. Yang, Macromolecules, 2001, 34, 4542.

22. K. Aou, S.L. Hsu, Macromolecules, 2006, 39, 3337.

23. J. Blomqvist, L.O. Pietila, B. Mannfors, Polymer, 2001, 42, 109.

24. J. Blomqvist, Polymer, 2001, 42, 3515.

25. T.T. Lin, X.Y. Liu, C. He, J. Phys. Chem. B, 2010, 114, 3133.

26. X. Yang, S. Kang, Y. Yang, K. Aou, S.L. Hsu, Polymer, 2004, 45, 4241.

27. J.L. Espartero, I. Rashkov, S.M. Li, N. Manolova, M. Vert, Macromolecules, 1996, 29, 3535. 
28. Hyperchem. HyperChem(TM) Molecular Modelling System, Release 4.5 SGI, Hypercube; Hyperchem(TM) Molecular Modelling System, Release 5.01 for Windows, Hypercube, Inc. (1998)

29. M.J Frisch, G.W. Trucks, H.B. Schlegel, G.E. Scuseria, M.A. Robb, J.R. Cheeseman, J. Montgomery, A.T. Vreven, K.N. Kudin, J.C. Burant, J.M. Millam, S.S. lyengar, J. Tomasi, V. Barone, B. Mennucci, M. Cossi, G. Scalmani, N. Rega, G.A, Petersson, H. Nakatsuji, M. Hada, M. Ehara, K. Toyota, R. Fukuda, J. Hasegawa, M. Ishida, T. Nakajima, Y. Honda, O. Kitao, H. Nakai, M. Klene, X. Li, J.E. Knox, H.P. Hratchian, J.B. Cross, V. Bakken, C. Adamo, J. Jaramillo, R. Gomperts, R.E. Stratmann, O. Yazyev, A.J. Austin, R. Cammi, C. Pomelli, J.W. Ochterski, P.Y. Ayala, K. Morokuma, G.A. Voth, P. Salvador, J.J. Dannenberg, V.G. Zakrzewski, S. Dapprich, A.D. Daniels, M.C. Strain, O. Farkas, D.K. Malick, A.D. Rabuck, K. Raghavachari, J.B. Foresman, J.V. Ortiz, Q. Cui, A.G. Baboul, S. Clifford, J. Cioslowski, B.B. Stefanov, G. Liu, A. Liashenko, P. Piskorz, I. Komaromi, R.L. Martin, D.J. Fox, T. Keith, M.A. AlLaham, C.Y. Peng, A. Nanayakkara, M. Challacombe, P.M.W. Gill, B. Johnson, W. Chen, M.W. Wong, C. Gonzalez, J.A. Pople, Gaussian 09, Gaussian, Inc., Wallingford CT, 2004. (2009)

30. I. Irsai, C. Majdik, A. Lupan, R. Silaghi-Dumitrescu. Journal of Mathematical Chemistry, 2011, 50(4), 703. 